\title{
FORRCART 2020, NUEVA VARIEDAD DE CÁRTAMO FORRAJERO EN MÉXICO
}

\author{
FORRCART 2020, A NEW FORAGE \\ SAFFLOWER VARIETY IN MEXICO
}

\begin{abstract}
Xochilt M. Ochoa-Espinoza ${ }^{1,5 *}$, Lope Montoya-Coronado', David G. Reta-Sanchez ${ }^{2}$, Alberto BorbónGracia', Néstor A. Aguilera-Molina', Eva Ávila-Casillas ${ }^{3}$, Carlos I. Cota-Barreras ${ }^{4}$ y Pedro Cano-Ríos ${ }^{5}$
\end{abstract}

\begin{abstract}
'Instituto Nacional de Investigaciones Forestales, Agrícolas y Pecuarias (INIFAP), Campo Experimental Norman E. Borlaug, Ciudad Obregón, Sonora México. ${ }^{2}$ INIFAP, Campo Experimental Delicias, Delicias, Chihuahua, México. ${ }^{3}$ INIFAP, Campo Experimental Valle de Mexicali, Mexicali, Baja California, México. ${ }^{4}$ INIFAP, Campo Experimental Todos Santos, La Paz, Baja California Sur, México. ${ }^{5}$ Universidad Autónoma Agraria Antonio Narro, Unidad Laguna, Torreón, Coahuila, México.
\end{abstract}

*Autor de correspondencia (ochoaxochilt@gmail.com)

El cártamo (Carthamus tinctorius L.) es una opción como forraje en diversas localidades de México (Montoya, 2010). Es una especie que puede ser cultivada en suelos con moderada tolerancia a salinidad (Maas, 2019) y con limitada disponibilidad de agua y tiene el potencial para ser cultivado en regiones áridas y semiáridas (Bar-Tal et al., 2008). El hecho de que el cártamo pueda progresar en ambientes de estrés por temperaturas extremas, sequías y salinidad ha facilitado su expansión en áreas de todo el mundo, donde las restricciones climáticas y del suelo han impedido producir cultivos alimenticios y convencionales (Hussain, et al., 2015). En trabajos realizados en Italia e Israel, los cultivares de cártamo sin espinas han mostrado buen potencial forrajero con rendimientos de 4.0 a $5.2 \mathrm{t}$ ha-1 de materia seca (MS) en la etapa de inicio de yemas florales (Landau et al., 2005), además de una composición química del forraje con contenidos de proteína cruda (PC) de $14.0 \%$, fibra detergente neutro (FDN) de $41.0 \%$ y fibra detergente ácida (FDA) de 30.0 \% (Danieli et al., 2011; Landau et al., 2005). En estudios realizados en Matamoros, Coahuila, México, los rendimientos de materia seca (MS) de cártamos tradicionales cosechados en inicio de botón floral fluctuaron de 5.1 a $7.7 \mathrm{t} \mathrm{ha}^{-1}$, su forraje presentó contenidos de PC de 16.2 a $19.7 \%$, FDN de 39.3 a $49.3 \%$, FDA de 33.8 a $43.4 \%$ y de 1.22 a $1.40 \mathrm{Mcal} \mathrm{kg}^{-1}$ de MS en energía neta para lactancia ( $E N_{\mathrm{L}}$ ) (Reta et al., 2015).

La utilización de cultivares de cártamo sin espinas para forraje ofrece la posibilidad de incrementar el rendimiento de MS al retrasar la cosecha durante el desarrollo de las yemas florales, conservando una composición nutrimental del forraje aceptable (Reta, et al., 2015). Con base en las necesidades de los productores de forraje, el programa de mejoramiento de cártamo del Instituto Nacional de
Investigaciones Forestales, Agrícolas y Pecuarias (INIFAP), inició el desarrollo de variedades con las características de alta producción y calidad forrajera.

El proceso para obtener la variedad de cártamo FORRCART 2020 inició en el Programa de Mejoramiento Genético de Cártamo del Campo Experimental Norman E. Borlaug del INIFAP, en el Valle del Yaqui, Sonora ( $27^{\circ} 22^{\prime} 14.39^{\prime \prime}$ latitud N, $109^{\circ}$ 55' 52.40" longitud O y 40 m de altitud). Proviene de la cruza entre la línea RUSSOS-R-262 y la línea SEL KINO-76. RUSSOS-R-262 es una línea con pocas espinas y alta tolerancia a roya (Puccinia carthami C.) y tizón de la hoja (Alternaria carthami C.). SEL KINO-76 es una línea seleccionada de la variedad Kino-76 con alta tolerancia a roya y pocas espinas. La población segregante se manejó con el método genealógico o de pedigrí. A partir de la línea experimental que se identificó con la genealogía CD868-A3-2-1-0Y se realizó selección masal en la generación $F_{1}$ y selección individual de $F_{2}$ hasta $F_{4}$. La selección en masa para la obtención de la línea avanzada se llevó a cabo en la generación $F_{5}$. A partir de $F_{6}$ se evaluó en ensayos de rendimiento forrajero y de calidad, los cuales se presentan en los Cuadros 1 y 2.

En cuanto a las características morfológicas referidas en los descriptores varietales de la Unión Internacional para la Protección de las Obtenciones Vegetales (UPOV, 1990) destacan las siguientes: presenta una altura en promedio de $151 \mathrm{~cm}$, su hábito de crecimiento es erecto, las hojas son de color verde claro, de forma ovoide, el dentado de la hoja es ausente o muy débil (Figura 1). El tallo es erecto, sólido y resistente al acame. La etapa de cosecha es en inicio de floración (EC61) (Flemmer et al., 2015), aproximadamente a los 95-105 días después de la siembra. Los pétalos son 
de color amarillo y cuando se secan no cambian de color.

La variedad FORRCART 2020 fue registrada oficialmente para su protección en julio de 2020 en el catálogo nacional de variedades vegetales (CNVV) con número de registro provisional 4105-CAR-009-060720/C del Servicio Nacional de Inspección y Certificación de Semillas (SNICS) perteneciente a la Secretaria de Agricultura y Desarrollo Rural (SADER). La semilla básica está a disposición de los productores de México en el CENEB-INIFAP, en Ciudad Obregón, Sonora.

\section{AGRADECIMIENTOS}

Al Instituto Nacional de Investigaciones Forestales Agrícolas y Pecuarias (INIFAP), a la Secretaría de Agricultura y Desarrollo Rural (SADER), a la Fundación PIEAES de Sonora, México, A. C. y al Patronato para la Investigación y Experimentación Agrícola del estado de Sonora, por el financiamiento de los trabajos de investigación que condujeron a la obtención de la variedad FORRCART 2020.

Cuadro 1. Rendimiento de materia seca (MS), proteína cruda $(\mathrm{PC})$ y energía neta para la lactancia $\left(E N_{L}\right)$ en tres cultivares de cártamo en la Comarca Lagunera.

\begin{tabular}{lllll}
\hline Variedad & $\mathrm{MS}\left(\mathrm{kg} \mathrm{ha}^{-1}\right)$ & $\mathrm{PC}\left(\mathrm{kg} \mathrm{ha}^{-1}\right)$ & $\mathrm{EN}_{\mathrm{L}}\left(\mathrm{Mcal} \mathrm{ha}^{-1}\right)$ & Espinas en el forraje \\
\hline FORRCART 2020 & 10,531 & 1,462 & 14,216 & Ausentes \\
GUAYALEJO & 8,360 & 1,368 & 11,453 & Presentes \\
PROMESA & 9,118 & 1,532 & 13,221 & Presentes \\
\hline
\end{tabular}

Cuadro 2. Proteína cruda (PC), fibra detergente acida (FDA) y fibra detergente neutra (FDN) en tres cultivares de cártamo en la Comarca Lagunera.

\begin{tabular}{lllll}
\hline Variedad & PC (\%) & FDA (\%) & FDN (\%) & $\mathrm{EN}_{\mathrm{L}}\left(\mathrm{Mcal} \mathrm{kg}^{-1} \mathrm{MS}\right)$ \\
\hline FORRCART 2020 & 13.8 & 37.4 & 44.5 & 1.35 \\
GUAYALEJO & 16.3 & 37.7 & 40.7 & 1.37 \\
PROMESA & 16.8 & 34.4 & 39.4 & 1.45 \\
\hline
\end{tabular}

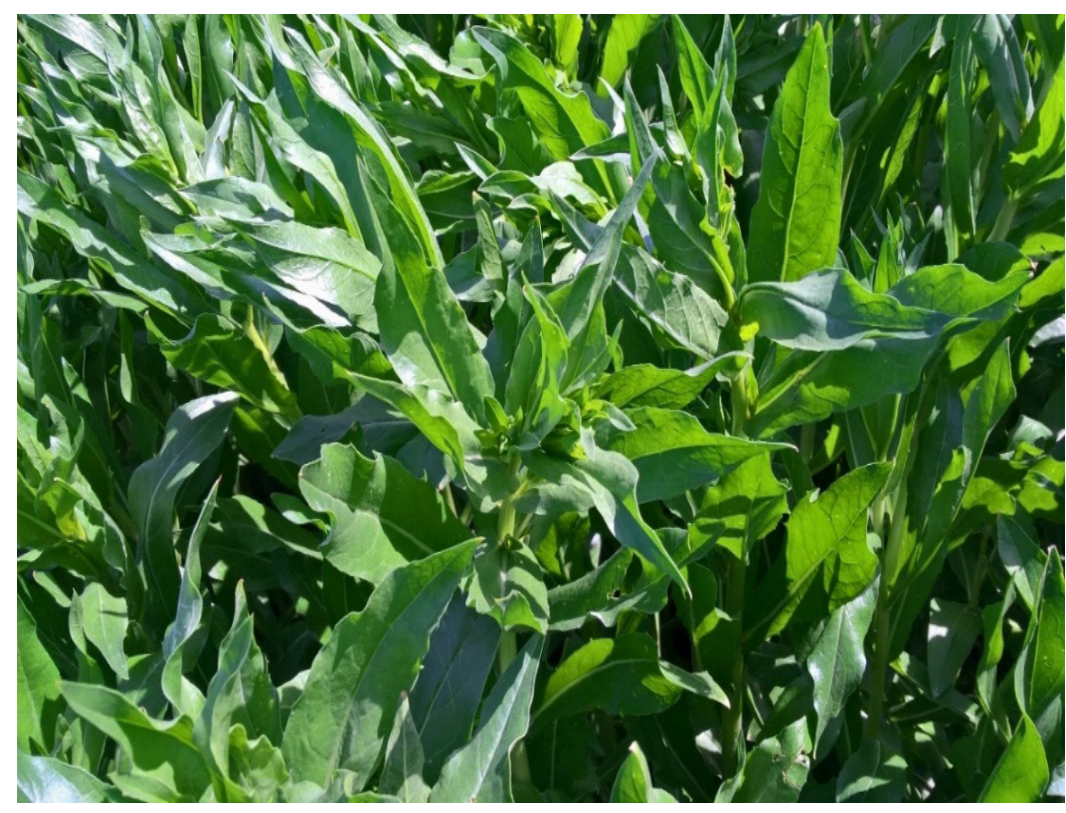

Figura 1. Variedad de cártamo forrajero FORRCART 2020 sin espinas. 


\section{BIBLIOGRAFÍA}

Bar-Tal A., S. Landau, Z. Li-xin, T. Markovitz, M. Keinan, L. Dvash, ... and Z. G. Weinberg (2008) Fodder quality of safflower across an irrigation gradient and with varied nitrogen rates. Agronomy Journal 100:1499-1505, https://doi.org/10.2134/agronj2007.0353

Danieli P. P., R. Primi, B. Ronchi, R. Ruggeri, F. Rossini, S. Del Puglia and C. F. Cereti (2011) The potential role of spineless saflower (Carthamus tinctorius L. var. inermis) as fodder crop in central Italy. Italian Journal of Agronomy 6:19-22, https://doi.org/10.4081/ija.2011. e4

Flemmer A. C., M. C. Franchini and L. I. Lindström (2015) Description of safflower (Carthamus tinctorius L.) phenological growth stages according to the extended BBCH scale. Annals of Applied Biology 166:331-339, https://doi.org/10.1111/aab.12186

Hussain M. I., D. A Lyra, M. Farooq, N. Nikoloudakis and N. Khalid (2015) Salt and drought stresses in safflower: a review. Agronomy for Sustainable Development 36:4, https://doi.org/10.1007/ s13593-015-0344-8
Landau S., G. Molle, N. Fois, S. Friedman, D. Barkai, M. Decandia, ... and M. Sitzia (2005) Safflower (Carthamus tinctorius L.) as a novel pasture species for dairy sheep in the Mediterranean conditions of Sardinia and Israel. Small Ruminant Research 59:239-249, https://doi.org/10.1016/j.smallrumres.2005.05.008

Maas E. V. (2019) Salt tolerance of plants. In: CRC Handbook of Plant Science in Agriculture. Vol. II. B. R. Christie (ed.). CRC Press. Boca Raton, Florida, USA. pp:57-66

Montoya C. L. (2010) El Cultivo del Cártamo (Carthamus tinctorius L.) en México. Talleres Gráficos SGI. Ciudad Obregón, Sonora, México. $96 \mathrm{p}$.

Reta S. D. G., J. S. Serrato C., A. Gaytán M., H. M. Quiroga G., G. Orozco H. y J A. Payán G. (2015) Potencial forrajero de variedades comerciales y genotipos élite de cártamo en la Comarca Lagunera. Agrofaz 15:27-38.

UPOV, International Union for the Protection of New Varieties of Plants (1990) Guidelines for the conduct of tests for distinctness, homogeneity and stability. Safflower (Carthamus tinctorius L.). TG/134/3. International Union for the Protection of New Varieties of Plants. Geneve, Suitzerland. 20 p. 